\title{
Inverse conduction problems and quantitative infrared thermography
}

\author{
by $A$. llyinsky
}

Moscow State University on Forestry, Dept. of Physics, Mitischi-1, Moscow Region, 141001, RUSSIA

\begin{abstract}
The efficiency of spectral methods of ICP solution for processing the IRT data has been demonstrated. The statistical analysis of nongaussian IRT noises using nonparametric Kolmogorov-Smimov statistics has been carried out and consistent estimation of cumulative probability function for the ITR noises has been found.

The nonconsistency of point estimation for optimal regularization parameter selection procedure for the case of nongaussian IRT noises has been shown.

The new selection rule for optimal regularization parameter value using nonparametric statistics of Smirnov-Kramer-von Mises ( $\omega^{2}$ - test) for the case of nongaussian IRT noises has been proposed.
\end{abstract}

\section{Inverse conduction problems and processing IR thermography data}

The measurement of non-uniform heat flux over conductive elements is a basic measuring operation in engineering. Any heat flux identification technique includes the transformation of heat flux field into temperature field, the temperature field estimation and the conversion of a temperature field into heat flux distribution by solving the appropriate inverse conduction problem (ICP). The thin metallic film technique is a rather popular method in aerospace applications [1] and for the small Biot number accurately converts a 2-D heat flux distribution into a 2-D temperature field. Recent progress in measurements of the spatial distributions of surface temperature by infrared thermography (IRT) creates a sound experimental base and excellent opportunities for further improvement of the heat transfer identification. The nondisturbing IRT provides us with experimental information on a 2-D temperature field at the enormous rate of $10^{7}-10^{8}$ Bytes/sec with highest spatial resolution. This feature of the IRT implies very strict restrictions on efficiency and rate of processing of temperature data in numerical solution of the ICP.

The ICP is the ill-posed problem according to the classification by Hadamard due to the discontinuity of the differential operator of Laplace and, hence, the ICP solutions lead to the tremendous amplification of the noise in measured temperature data. To solve ICP the special procedures called regularization of the ICP have been developed [2]. The essence of regularization is the approximation of noncontinuos operators by a family of continuous operators and the proper selection of the regularization parameter using given a priori information on the inverse problem. When the regularization parameter decreases, approximated operator approaches to the noncontinuous operator and noise in solution increases faster. From another hand, the increasing regularization parameter increases the smoothness of solution, but the accuracy of approximation in general becomes worse. This dilemma between accuracy and smoothness should be solved by the appropriate choosing of regularization parameter. The procedure of selection of the regularization parameter is based on the analysis of available a priori information on the solution and the processed data. For that reason, the additional a priori information on the accuracy of temperature measurements should be at hand to find a regularized solution of the ICP. Also it is necessary to construct a rule for proper selection of the value of the parameter of regularization to achieve the best possible solution of the ICP for a given a priori information and accuracy of IRT measurements.

The existing methods for solution of the ICP could be classifled into following groups.

- The step regularization methods. 
The ICP solution by direct numerical differentiation of experimental data with noise is the fastest and the simplest one. This technique, however, requires an implicit step regularization procedure and could not be used for processing of data obtained on fine grids [1].

- The variational methods.

The most general and popular approach in ICP solutions is a classic variational technique developed by Tichonov [2] and extensively studied by Alifanov [3]. The system of differential equation is replaced by the functional minimizing difference between the integral solution of the direct conduction problem $\mathbf{T}(\widetilde{\mathbf{q}})$ and measured data $\{\mathbf{T}\}$ with an additional functional called stabilizer in the form

$$
\mathbf{J}(\widetilde{\mathbf{q}})=\int_{\mathrm{s}}[\mathbf{T}(\widetilde{\mathbf{q}})-\{\mathbf{T}\}] \mathbf{d S}+\alpha \Omega(\widetilde{\mathbf{q}})
$$

The heat flux distribution is calculated as

$$
\widetilde{\mathbf{q}}=\arg \min \mathbf{J}(\widetilde{\mathbf{q}})
$$

The minimization of functional transforms the solution of the initial ICP into the solution of the system of linear algebraic equations. However, the regularized solution depends on the right selection of the stabilizer $\Omega$. To identify the $\mathrm{N}$-dimensional vector of heat flux by variational methods approximately $\mathrm{N}^{3}$ operations should be executed.

- The iterational methods.

The more advanced method of ICP solution is the iterative technique developed by Alifanov [3]. This technique is based on minimization of functional constructed on the difference between exact solution and measured data $\{T\}$ in the form

$$
\mathbf{J}(\widetilde{\mathbf{q}})=\int_{s}[\mathbf{T}(\widetilde{\mathbf{q}})-\{\mathbf{T}\}] \mathbf{d} \mathbf{S}+\alpha \Omega(\widetilde{\mathbf{q}})
$$

by using iterational sequences as

$$
\widetilde{\mathbf{q}}_{\mathbf{k}+1}=\widetilde{\mathbf{q}}_{\mathbf{k}}-\boldsymbol{\beta}_{\mathbf{k}} \mathbf{J}^{\prime}\left(\widetilde{\mathbf{q}}_{\mathbf{k}}\right) \quad \mathbf{J}\left(\tilde{\mathbf{q}}_{\mathbf{k}+1}\right)<\mathbf{J}\left(\tilde{\mathbf{q}}_{\mathbf{k}}\right)
$$

with $k=0,1,2, \ldots m$. The iterational process is stopped after the $m$-th iteration. The number of iterations $m$ should be considered as the regularization parameter. The complex adjoint conduction problem should be determined and retrospectively solved to find the gradient of the functional. The iterational methods allow to reduce the total number of operations to $\mathrm{mN}^{2}$.

- The spectral methods

Recently the new spectral technique for non-uniform heat flux identification has been proposed [4]. The method is based on the expansion of heat flux and temperature in Fourier series on eigenfunctions of discrete Laplacian over a rectangular mesh and Wiener filtration of temperature data as a precondition procedure. The projection subspace dimension is used as a regularization parameter. The two-point heat sources at distance of $2 \mathrm{~mm}$ have been resolved by spectral method on a copper plate with $0.85 \mathrm{~mm}$ thickness [5]. The usage of an efficient 2-D sine FFT and IFFT for implementation expansions in Fourier series drastically decreases the number of computational operations to $\mathrm{N} \mathrm{og}_{2} \mathrm{~N}$. This feature provides the spectral algorithm with a high processing rate potential and creates opportunities for its realization in real time mode.

To process $256 \times 512$ pixels of $I R$ frame it is necessary to complete $10^{15}, 10^{11}$ and $10^{6}$ operations applying variational, iterational and spectral methods respectively. For that reason the spectral technique is most suitable for processing IRT data.

The selection rule for subspace dimension has been proposed [4] for the case of gaussian noises in temperature measurements using the point estimation technique. However, for nongaussian errors the application of the point estimation technique usually leads to inconsistency and inefficiency.

In present paper nonparametric statistics for regularization criteria in spectral methods is studied and a new selection rule for optimal subspace dimension is proposed.

\section{Spectral method for IRT data processing}

Let us consider the spectral method for solution of ICP for IRT data acquired on a square, over thin metal film, given in dimensionless form. The set of pixels forms a rectangular mesh with $(\mathrm{N}+1)^{2}$ nodes $\left\{X_{i}, Y_{j}\right\}$ and step $h=1 / N$ on the unit square $0 \leq X \leq 1 ; 0 \leq Y \leq 1$. The dimensionless energy balance for each internal pixel can be written as 


$$
\begin{aligned}
& \frac{d T_{i, j}(t)}{d t}=\Delta^{h} T_{i, j}(t)+q_{i, j}(t) \\
& \Delta^{h} T_{i, j}=\left(T_{i, j+1}+T_{i, j-1}+T_{i+1, j}+T_{i-1, j}-T_{i, j}\right) / h^{2}
\end{aligned}
$$

with zero initial condition and boundary conditions.

The eigenfunctions of a spectral problem for mesh Laplacian $\Delta^{h}$ on the unit square form a complete orthonormal basis in the space of mesh function as

$$
\mathbf{u}_{1, j}^{k, m}=2 \sin (k \pi i h) \sin (m \pi j h)
$$

with eigenvalues

$$
\lambda^{k, m}\left(\Delta^{\mathrm{h}}\right)=4 \mathrm{~h}^{-2}\left\{\sin ^{2}(\mathrm{k} \pi \mathrm{h} / 2)+\sin ^{2}(\mathrm{~m} \pi \mathrm{h} / 2)\right\}
$$

where $\mathbf{0}<\mathbf{k}, \mathbf{m}<\mathbf{N}$ are integers

Expanding the solution in a Fourier

$$
\mathbf{T}_{i, j}=\sum_{\mathrm{l}=0}^{\mathrm{N}-1} \sum_{\mathrm{j}=0}^{N-1} \mathbf{T}^{\mathrm{k}, \mathrm{m}} \mathbf{u}_{\mathrm{i}, \mathrm{j}}^{\mathrm{k}, \mathrm{m}} \mathbf{q}_{\mathrm{i}, \mathrm{J}}=\sum_{\mathrm{i}=0}^{\mathrm{N}-1} \sum_{\mathrm{j}=0}^{N-1} \mathbf{q}^{k, \mathrm{~m}} \mathbf{u}_{\mathrm{i}, \mathrm{j}}^{\mathrm{k}, \mathrm{m}}
$$

we simplify the finite-difference balance equations (5) to the diagonal form

$$
\frac{d T^{k, m}(t)}{d t}=-\lambda^{k, m} T^{k, m}(t)+q^{k, m}(t)
$$

with zero initial conditions.

The solution of the balance equation in the diagonal form (9) can be written in the explicit form as a convolution integral

$$
T^{k, m}(t)=\int_{0}^{k} q^{k, m}(\xi) \exp \left(-\lambda^{k, m}(t-\xi)\right) d \xi
$$

To solve the Fredholm integral equation (10) we can use a powerful deconvolution method [2]. However, for quasi-steady-state conditions the integral equation (10) is simplified to

$$
q^{k, m}(t)=\lambda^{k, m} T^{k, m}(t)
$$

After calculation of components $q^{\mathbf{k}, \mathbf{m}}$ we can construct a heat flux distribution in the space domain using the Fourier expansion (8).

Unfortunately, the direct application of spectral methods is an unstable procedure due to the tremendous amplification of high frequency noise in IRT data. To improve the stability of spectral methods, Fourier components $\left\{\mathbf{T}^{\mathbf{k}, m}(t)\right\}$ are filtered by an appropriate 2-D low pass filter with frequency response $\mathbf{w}^{\mathbf{k}, \mathbf{m}}$. The regularization parameter of a 2-D low pass filter is selected using a priori information on temperature measurements to increase a conditionness of the algorithm.

Let the IRT data $\left\{\mathbf{T}_{\mathbf{i}, \mathbf{j}}\right\}$ on mesh $\mathbf{D}_{\mathbf{h}}$ contain some measurement errors $\mathbf{E}_{\mathbf{i}, \mathbf{j}}$ :

$$
\left\{T_{\mathbf{i}, \mathbf{j}}\right\}=\mathbf{T}_{\mathbf{i}, \mathbf{j}}+\mathbf{E}_{\mathbf{i}, \mathbf{j}}
$$

Then the accuracy of the developed heat flux estimation technique is defined as

$$
\delta \widetilde{\mathbf{q}}_{i, j}=\sum_{k=1}^{N-1} \sum_{m=1}^{N-1} \lambda^{k, m}\left(W^{k, m}-1\right) T^{k, m} u_{i, j}^{k, n}+\sum_{k=1}^{N-1} \sum_{m=1}^{N-1} \lambda^{k, m} W^{k, m} \mathbf{E}^{k, m} u_{i, j}^{k, n}
$$

The first term defines an approximation error due to filtration and the second term presents a noise amplification error. The preprocessing filtration of initial data may be considered as a regularization procedure with the filter bandwidth as a regularization parameter. The decrease of a filter bandwidth causes less noise amplification but higher approximation error and vice versa, the enlargement of a bandwidth reduces an approximation error and magnifies high frequency noise. The proper selection of the filter bandwidth is based on additional criteria which could be derived from available a priori information.

The quasi-optimal filter is implemented as a projection onto a subspace with dimension $\mathbf{M}^{2}$ with a 2-D Hamming window to reduce an effect of rippling as 


$$
\begin{aligned}
& \left(W^{k, m}(M)\right)=0.54+0.46 \cos \left(\pi\left(k^{2}+m^{2}\right)^{1 / 2} / M\right) \text { if }\left(k^{2}+m^{2}\right)^{1 / 2} / M \leq 1 \\
& \left(W^{k, m}(M)\right)=0 \\
& \text { if }\left(k^{2}+m^{2}\right)^{1 / 2} / M>1
\end{aligned}
$$

To determine a quasi-optimal filtration, we must define a cutoff frequency or dimension of subspace $\mathbf{M}$.

The only procedure based on a statistical analysis of the difference between initial noisy temperature IRT data $\{\mathbf{T}\}$ and temperature data after filtration $\mathbf{T}^{F}$, referred as the temperature residue $\mathbf{T}^{\mathrm{R}}$, with a priori information on a temperature measurement accuracy can provide a stable and reliable result.

$$
\mathbf{T}_{i, j}{ }^{R}=\left\{T_{i, j}\right\}-T_{i, j}{ }^{p}=\sum_{i=0}^{N-1} \sum_{j=0}^{N-1}\left\{T^{k, m}\right\}\left(1-W^{k, m}(M)\right) \mathbf{u}_{1, j}^{k, m}
$$

\section{Nonparametric statistics as regularization criteria for ICP}

In a previous paper [4], for the case of gaussian noises in IRT data, the simple rule for optimal dimension selection of $\mathbf{M}$ based on point estimation has been proposed

$$
M=\arg \min \left(\left|\sigma(E)-\sigma\left(T^{R}\right)\right|\right)
$$

where $\sigma(\mathbf{Z})$ - - is the standard deviation of random variable $\mathbf{Z}$.

Let us analyze the statistical properties of IRT noises reported earlier [5]. The IRT data have been obtained by AVIO Thermal Video System TVS 2000ST (Nippon Avionics, Japan) on an isothermal copper plate at room temperature. First of all it is necessary to carry out the normality tests on the noise of IRT data using the Kolmogorov-Smimov (K-C) criteria. Let us sort in ascending order $32 \times 32$ pixels IRT noise $\mathbf{E}_{\mathrm{ij},}$, transform data into variational series (the order statistics) $\mathbf{E}_{\mathbf{i}}{ }^{\mathbf{s}}$

$$
\mathbf{E}_{\mathrm{ij}} \rightarrow \mathbf{E}_{\mathrm{i}}^{\mathrm{S}}: \mathbf{E}_{\mathrm{i}} \geq \mathbf{E}_{\mathrm{j}-\mathrm{1}}
$$

and calculate the empirical cumulative probability function as

$$
\begin{aligned}
& \mathbf{F}(\mathbf{E})=0 \quad \text { if }-\infty<\mathbf{E}<\mathbf{E}_{1}^{s} \\
& \mathbf{F}(\mathbf{E})=i / n \text { if } \mathbf{E}_{\mathrm{i}}^{s} \leq \mathbf{E}<\mathbf{E}_{i+1}{ }^{s} \\
& \mathbf{F}(\mathbf{E})=1 \text { if } \mathbf{E}_{\mathbf{n}}{ }^{s} \leq \mathbf{E}<\infty
\end{aligned}
$$

which is compared with normal distribution $N\left(\mu, \sigma^{2}\right)$ in the form

$$
\mathbf{F}_{\text {NORM }}(\mathbf{E})=\operatorname{erf}((\mathbf{E}-\boldsymbol{\mu}) / \sigma)
$$

Figure 1 shows the comparison of the empirical probability function (18) with the normal distribution (19). The K-C test is based on nonparametric statistics D [6]

$$
\mathbf{D}=\max \left(\left|\mathbf{F}(\mathbf{E})-\mathbf{F}_{\text {NORM }}(\mathbf{E})\right|\right)
$$

According to $\mathrm{K}-\mathrm{C}$ test with significance level less than 0.01 , the hypothesis of normality for IRT data should be rejected. Moreover, the K-C test provides us with the powerful tool to find an adequate approximation for empirical cumulative function. For example the empirical data in Figure 1 could be adequate fitted as sum of two gaussian curves by the following expression

$$
F_{\text {APpR }}(E)=0.354 \text { erf }((E-\mu) / 0.301)+0.646 \mathrm{erf}((E-\mu) / 0.646)
$$

Let us consider the heat flux restoration by a spectral technique using IRT data with nongaussian noises. The initial heat flux distribution with two anisotropic peaks over a conductive plate $\left(L_{x}=L_{y}=0.1 \mathrm{~m}, d=1 \mathrm{~mm}, k=15 \mathrm{~W} / \mathrm{mK}\right.$ ) is presented in Figure 2. IRT data is deteriorated by equal mixture of two gaussian noises with zero mean and different standard deviations $\sigma_{1}=0.11 \mathrm{~K}$ and $\sigma_{2}=0.22 \mathrm{~K}$. Figure 3 displays the $16 \times 16$ pixel IRT data over the conductive wall with zero boundary conditions and adiabatic internal surface. The two rather sharp peaks in heat flux distributions have been converted into one smooth peak in temperature distribution due to the conduction effects. Using the spectral technique with quasi-optimal filtration (14) as a regularization procedure, the temperature residue $T^{R}$ and relative heat flux residue $\mathbf{q}^{\mathbf{R}}=(\mathbf{q}-\tilde{\mathbf{q}}) / \mathbf{q}_{\text {MAX }}$ have been calculated for a different subspace dimension $\mathbf{M}$. To estimate the optimal value of subspace dimension $\mathbf{M}$, the Smirnov-Kramer-von Mises criterion $\left(\omega^{2}\right.$-test) has been used in the form [6] 


$$
\omega^{2}(M)=\int_{0}\left[F(E)-F\left(T^{R}(M)\right)\right]^{2} d F(E)
$$

The values of $\sigma\left(\mathbf{T}^{R}\right), \sigma\left(\mathbf{q}^{R}\right)$ and $\omega^{2}$ for different $\mathbf{M}$ are presented in table 1 .

Table 1. The effect of subspace dimension $\mathbf{M}$ on statistical characteristics of temperature and heat flux residues.

\begin{tabular}{|cccccccc|}
\hline $\mathbf{M}$ & 4 & 6 & 7 & 8 & 9 & 10 & 12 \\
$\sigma\left(\mathbf{T}^{\mathrm{R}}\right)$ & 14.8 & 9.8 & 7.9 & 7.3 & 6.9 & 6.5 & 6.1 \\
$\sigma\left(\mathbf{q}^{\mathrm{R}}\right) 10^{2}$ & 4.4 & 2.5 & 1.9 & 1.6 & 1.6 & 1.7 & 2.2 \\
$\boldsymbol{\sigma}^{2} 10^{4}$ & 61 & 32 & 29 & 35 & 42 & 50 & 61 \\
\hline
\end{tabular}

The comparison of values of $\sigma\left(\mathrm{T}^{\mathrm{R}}\right)$ from Table 1 and $\sigma(\mathbf{E})=5.5$ demonstrates the impossibility of applying point estimation rule (16) for proper selection of subspace dimension $\mathbf{M}$ for nongaussian IRT noises.

For nongaussian IRT noises the new criteria of selection of subspace dimension $\mathbf{M}$ is constructed in form of nonparametric $\omega^{2}$ statistic

$$
M=\arg \min \left(\omega^{2}(M)\right)
$$

For a given IRT noise the rule (23) provides us with the value $\mathbf{M}=7$, however, the minimum of the standard deviation of heat flux residue $\sigma\left(q^{R}\right)$ takes place at $\mathbf{M}=9$. The heat flux distribution restored by a spectral method using nonparametric statistic (23) is presented in Figure 4. Nonparametric criteria for $\Phi^{2}$ allow to get a robust estimation of the optimal subspace dimension and a more smooth regularized solution of ICP. Generally, the proposed selection criteria (23) are less sensitive to the special form of the cumulative probability function of IRT noise.

\section{Conclusions}

The efficiency of spectral methods of ICP solution for processing IRT data has been shown.

The statistical analysis of nongaussian IRT noises using nonparametric Kolmogorov-Smimov statistics has been carried out and consistent estimation of cumulative probability function for the ITR noises has been found.

The nonconsistency of point estimation for optimal regularization parameter selection procedure for the case of nongaussian IRT noises has been shown.

The new selection rule for optimal regularization parameter value using nonparametric statistics of Smimov-Kramer-von Mises ( $\omega^{2}$-test) for the case of nongaussian IRT noises has been proposed. The results of numerical solution of IRT by spectral method with nongaussian noises demonstrates robustness and efficiency of the proposed technique.

\section{REFERENCES}

[1] Dumoulin J., Reulet P., Grenier P., Plazanet M., Millan P. Steady and Unsteady Wall Heat Transfer Mapping by Active Infrared Thermography, In Quantitative InfraRed Thermography, Ed. Balageas. Paris 1995, pp158-165.

[2] Tichonov A.N., Arsenin Ya., Solution of III-Posed Problems, Halsted, NY, 1977.

[3] Alifanov O.M. The Inverse Problem in Heat Transfer, Mashinostroenie, Moscow,1988. (in Russian)

[4] Ilyinsky A.I. - Net heat flux identification using digitized temperature data in reactor accidents with hot spot formation on thin wall with variable thermal conductivity - Proc. Int. Seminar on Heat and Mass Transfer in Severe Reactor Accidents, May 1995, Cesme, Turkey, 1995.

[5] llyinsky A.I. Ranieri S., Farina A., Pagliarini G. - New experimental technique for enhancement of spatial resolution in heat transfer measurements - accepted by 4th World Conference on Experimental Heat Transfer, Fluid Mechanics and Thermodynamics, Brussels, June, 1997.

[6] Lehmann E.L. Testing Statistical Hypotheses, Wiley, 1959. 


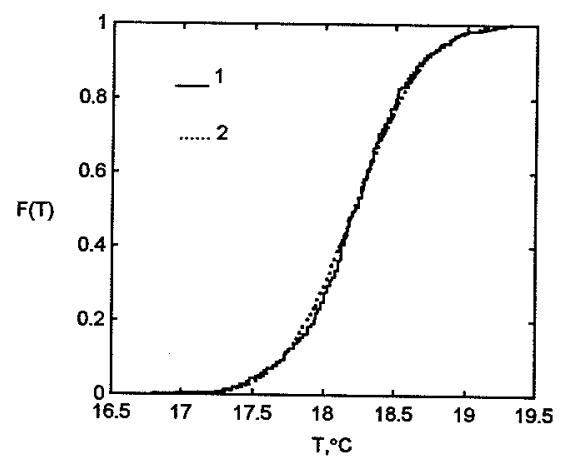

Figure 1. Cumulative probability function for IRT noise data (1) and gaussian error function (2).

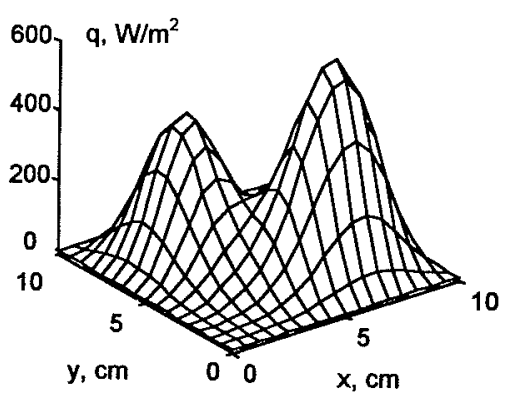

Figure 2. The exact heat flux distribution over conductive plate

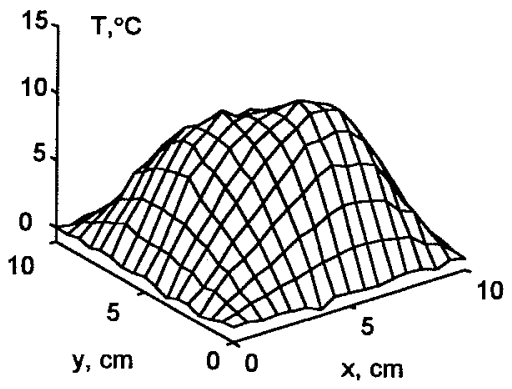

Figure 3. The IRT data over conductive plate with nongaussian noises

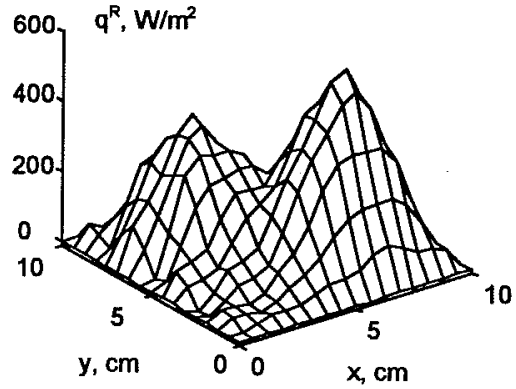

Figure 4. The restored heat flux distribution over conductive plate using spectral method for $M=7$. 